\title{
On the degree of MIMO systems
}

\author{
P. P. Vaidyanathan \\ Dept. of Electrical Engineering \\ California Institute of Technology \\ Pasadena, CA 91125 \\ Email:ppvnath@systems.caltech.edu
}

\begin{abstract}
MIMO channels and wireless communications systems have generated a great deal of renewed interest in linear system theory. This paper presents two results. The first is a simple proof based on first principles, of the known fact that the McMillan degree of a causal $M \times M$ MIMO system is at least as large as the degree of its determinant. The second is a new result which shows that the degree of the $M \times M$ system $z^{-1} \mathbf{G}(z)$ is equal to the degree of $\mathbf{G}(z)$ plus $M$ if and only if the causal system $\mathbf{G}(z)$ has an anticausal inverse. ${ }^{1}$
\end{abstract}

\section{INTRODUCTION}

MIMO channels and wireless communications systems have generated a great deal of renewed interest in linear system theory [1], [3], [4]. Even though MIMO system theory is a very well-established field [2], certain specific questions that come up in the context of communications require revisiting the fundamentals in order to generate new results.

In this paper we consider two basic questions for discrete time MIMO LTI systems. The first question, addressed in Sec. II, is on the relation between the McMillan degree or simply the degree of a system [2], [5], defined to be the minimum number of delays required in its implementation, and the degree of the determinant. The second question, addressed in Sec. III, is on the degree of a composite system formed by cascading an LTI system with a simple delay element.

\section{SYSTEM DEGREE AND DETERMINANT DEGREE}

Consider an $M \times M$ causal system

$$
\mathbf{H}(z)=\mathbf{h}(0)+\mathbf{h}(1) z^{-1}+\mathbf{h}(2) z^{-2}+\ldots,
$$

possibly IIR. For such a system it is well-known that

$$
\operatorname{deg}[\operatorname{det} \mathbf{H}(z)] \leq \operatorname{deg} \mathbf{H}(z) .
$$

This is usually proved by invoking fairly deep results such as, for example, the Smith-McMillan decomposition [2], [5]. In this section we give an elementary proof based on first principles. We divide the discussion into two cases.

\footnotetext{
${ }^{1}$ Work supported in parts by the Office of Naval Research grant N00014-06-1-0011 and the California Institute of Technology.
}

\section{II.1. Case when zeroth coefficient is zero}

First consider the special case where

$$
\mathbf{H}(z)=\mathbf{h}(1) z^{-1}+\mathbf{h}(2) z^{-2}+\ldots,
$$

that is, a system with constant term $\mathbf{h}(0)=\mathbf{0}$. Let $(\mathbf{A}, \mathbf{B}, \mathbf{C}, \mathbf{D})$ be a minimal realization of $\mathbf{H}(z)$. Since $\mathbf{h}(0)=\mathbf{0}$, we have $\mathbf{D}=\mathbf{0}$ so that

$$
\mathbf{H}(z)=\mathbf{C}(z \mathbf{I}-\mathbf{A})^{-1} \mathbf{B} .
$$

Denoting the degree of $\mathbf{H}(z)$ as $N$, the matrix $\mathbf{A}$ has size $N \times N$. We have three possible situations.

1) Case 1. $M=N$. In this case $\mathbf{B}$ and $\mathbf{C}$ are $N \times N$ matrices, and we have

$\operatorname{det} \mathbf{H}(z)=\frac{[\operatorname{det} \mathbf{C}][\operatorname{det} \mathbf{B}]}{\operatorname{det}(z \mathbf{I}-\mathbf{A})}=\frac{[\operatorname{det} \mathbf{C}][\operatorname{det} \mathbf{B}]}{a_{0}+a_{1} z+\ldots+a_{N} z^{N}}$

where $a_{0}+a_{1} z+\ldots+a_{N} z^{N}$ is the characteristic polynomial of $\mathbf{A}$. This shows that the determinant of $\mathbf{H}(z)$ has degree equal to the degree $N$ of the system $\mathbf{H}(z)$, unless $\mathbf{B}$ or $\mathbf{C}$ is singular (in which case $\operatorname{det} \mathbf{H}(z)=0$, and $\operatorname{deg}[\operatorname{det} \mathbf{H}(z)]=0$ ). This proves (1) for $M=N$.

2) Case 2. $M>N$. In this case the $M \times N$ matrix $\mathbf{C}$ has rank less than $M$. The determinant of the $M \times M$ matrix in Eq. (2) is therefore zero for all $z$, and Eq. (1) is trivially satisfied.

3) Case 3. $M<N$. In this case let us define the $N \times N$ system

$$
\mathbf{F}(z)=\left[\begin{array}{cc}
\mathbf{H}(z) & \mathbf{0} \\
\mathbf{0} & z^{-1} \mathbf{I}_{N-M}
\end{array}\right]
$$

This system continues to be a causal system with constant coefficient $\mathbf{f}(0)=\mathbf{0}$. Since it is a square matrix, it falls under Case 1 , so it follows that

$$
\operatorname{deg}[\operatorname{det} \mathbf{F}(z)] \leq \operatorname{deg} \mathbf{F}(z)
$$

From the definition (3) it is clear that

$$
\operatorname{deg}[\operatorname{det} \mathbf{F}(z)]=\operatorname{deg}[\operatorname{det} \mathbf{H}(z)]+N-M,
$$

assuming $[\operatorname{det} \mathbf{H}(z)] \neq 0$ (if this assumption is not true then Eq. (1) holds trivially). It also follows from Eq. (3) that

$$
\operatorname{deg}[\mathbf{F}(z)]=\operatorname{deg}[\mathbf{H}(z)]+N-M
$$


because $z^{-1} \mathbf{I}_{N-M}$ represents an independent system with $N-M$ inputs and outputs with a transfer function $z^{-1}$ between the $k$ th input and $k$ th output. By using Eq. (5) and (6) in Eq. (4), the desired result (1) follows immediately.

\section{II.2 Case when zeroth coefficient is nonzero}

Consider the $M \times M$ system

$$
\begin{aligned}
\mathbf{H}(z) & =\mathbf{h}(1) z^{-1}+\mathbf{h}(2) z^{-2}+\ldots \\
& =z^{-1} \underbrace{\left(\mathbf{h}(1)+z^{-1} \mathbf{h}(2)+\ldots\right)}_{\text {call this } \mathbf{G}(\mathrm{z})}
\end{aligned}
$$

Here $\mathbf{G}(z)=\sum_{n=0}^{\infty} \mathbf{g}(n) z^{-n}$ is a causal system with $\mathbf{g}(0)=\mathbf{h}(1)$ possibly nonzero. Since

$$
\mathbf{H}(z)=z^{-1} \mathbf{G}(z),
$$

it follows that

$$
\operatorname{det} \mathbf{H}(z)=z^{-M}[\operatorname{det} \mathbf{G}(z)] .
$$

Thus

$$
\operatorname{deg}[\operatorname{det} \mathbf{H}(z)]=M+\operatorname{deg}[\operatorname{det} \mathbf{G}(z)]
$$

unless [det $\mathbf{G}(z)$ ] is identically zero for all $z$ (in which case Eq. (1) is trivially true anyway). From Eq. (7) we also see that

$$
\operatorname{deg} \mathbf{H}(z)=\operatorname{deg}\left[z^{-1} \mathbf{G}(z)\right] \leq M+\operatorname{deg} \mathbf{G}(z)
$$

because we can always build $\mathbf{H}(z)$ from $\mathbf{G}(z)$ by cascading one delay to each output terminal. Thus we have

$$
\begin{aligned}
M+\operatorname{deg}[\operatorname{det} \mathbf{G}(z)] & =\operatorname{deg}[\operatorname{det} \mathbf{H}(z)] \quad(\text { from (9)) } \\
& \leq \operatorname{deg} \mathbf{H}(z) \quad(\text { proved, Sec. II.1) } \\
& \leq M+\operatorname{deg} \mathbf{G}(z) \quad(\text { from (10)) }
\end{aligned}
$$

which shows that

$$
\operatorname{deg}[\operatorname{det} \mathbf{G}(z)] \leq \operatorname{deg} \mathbf{G}(z)
$$

Since $\mathbf{G}(z)$ has unrestricted $\mathbf{g}(0)$, the inequality (11) has therefore been established for arbitrary causal $M \times M$ systems.

\section{SYSTEM CASCADED WITH DELAYS}

Let $\mathbf{G}(z)$ represent an $M \times M$ casual system with degree $N$. Define

$$
\mathbf{H}(z)=z^{-1} \mathbf{G}(z)
$$

We can certainly implement $\mathbf{H}(z)$ as a cascade of $\mathbf{G}(z)$ with $M$ delays, thus requiring a total of $N+M$ delays (Fig. 1). But is this the minimum number of delays? Surprisingly, the answer depends entirely on whether $\mathbf{G}(z)$ has an anticausal inverse or not, as we shall show (Theorem 1).

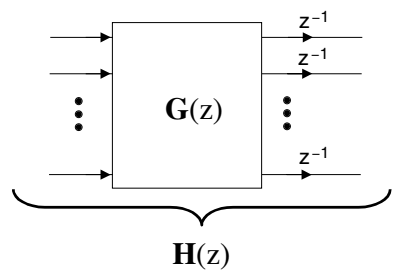

Figure 1. A cascade of $\mathbf{G}(z)$ with $z^{-1} \mathbf{I}$.

It is obvious from Fig. 1 that

$$
\operatorname{deg} \mathbf{H}(z) \leq N+M .
$$

To show that equality may not always hold, consider the example

$$
\mathbf{G}(z)=\left[\begin{array}{ll}
z^{-1} & z^{-1} \\
z^{-1} & z^{-1}
\end{array}\right]
$$

Even though there are many delays in this expression, this can be rewritten as

$$
\mathbf{G}(z)=z^{-1}\left[\begin{array}{l}
1 \\
1
\end{array}\right]\left[\begin{array}{ll}
1 & 1
\end{array}\right]
$$

so that the system can be implemented with one delay as shown in Fig. 2(a). So the degree is unity $(N=1)$. The cascaded system

$$
\mathbf{H}(z)=z^{-1} \mathbf{G}(z)=\left[\begin{array}{cc}
z^{-1} & 0 \\
0 & z^{-1}
\end{array}\right] \mathbf{G}(z)
$$

can certainly be implemented by adding two delays to represent the diagonal matrix (Fig. 2(b)) so that there are $N+M=3$ delays. But it is clear that we can rearrange the system as

$$
\mathbf{H}(z)=z^{-1} \mathbf{G}(z)=z^{-2}\left[\begin{array}{l}
1 \\
1
\end{array}\right]\left[\begin{array}{ll}
1 & 1
\end{array}\right]
$$

which only requires two delays (Fig. 2(c)). So the degree is $2<N+M$ in this case.
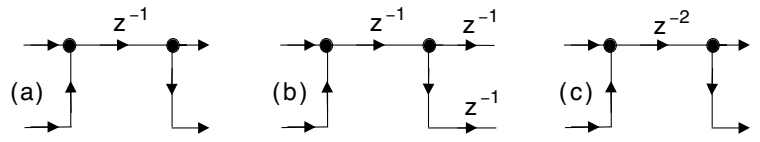

Figure 2. (a) Example of a system $\mathbf{G}(z)$ with degree one, (b) the cascaded system $\mathbf{H}(z)=z^{-1} \mathbf{G}(z)$ with two extra delays, and (c) structure for $\mathbf{H}(z)$ with mimimum number of delays.

To make progress towards Theorem 1, let $\left(\mathbf{A}_{1}, \mathbf{B}_{1}, \mathbf{C}_{1}, \mathbf{D}_{1}\right)$ represent a minimal realization of $\mathbf{G}(z)$. A structure for $\mathbf{H}(z)$ containing $N+M$ delays is shown in Fig. 3. Here the state vector $\mathbf{x}_{1}(n)$ has size $N$, and $\mathbf{x}_{2}(n)$ has size $M$ so that the 
state vector of the entire system:

$$
\mathbf{x}(n)=\begin{gathered}
N \\
\ldots \\
M
\end{gathered}\left(\begin{array}{c}
\mathbf{x}_{1}(n) \\
\ldots \ldots \ldots \\
\mathbf{x}_{2}(n)
\end{array}\right)
$$

has size $N+M$. The implementation in Fig. 3 is minimal if and only if it is reachable and observable [2], [5]. To check this, let us first write the state space equations and identify the $(\mathbf{A}, \mathbf{B}, \mathbf{C}, \mathbf{D})$ matrices of the cascade. We have

$$
\begin{aligned}
\mathbf{x}_{1}(n+1) & =\mathbf{A}_{1} \mathbf{x}_{1}(n)+\mathbf{B}_{1} \mathbf{u}(n) \\
\mathbf{x}_{2}(n+1) & =\mathbf{C}_{1} \mathbf{x}_{1}(n)+\mathbf{D}_{1} \mathbf{u}(n) \\
\mathbf{y}(n) & =\mathbf{x}_{2}(n)
\end{aligned}
$$

which can be rearranged into the form

$$
\begin{gathered}
\underbrace{\left[\begin{array}{c}
\mathbf{x}_{1}(n+1) \\
\mathbf{x}_{2}(n+1)
\end{array}\right]}_{\mathbf{x}(n+1)}=\underbrace{\left[\begin{array}{ll}
\mathbf{A}_{1} & \mathbf{0} \\
\mathbf{C}_{1} & \mathbf{0}
\end{array}\right]}_{\mathbf{A}} \underbrace{\left[\begin{array}{l}
\mathbf{x}_{1}(n) \\
\mathbf{x}_{2}(n)
\end{array}\right]}_{\mathbf{x}(n)}+\underbrace{\left[\begin{array}{l}
\mathbf{B}_{1} \\
\mathbf{D}_{1}
\end{array}\right]}_{\mathbf{B}} \mathbf{u}(n) \\
\mathbf{y}(n)=\underbrace{\left[\begin{array}{ll}
\mathbf{0} & \mathbf{I}
\end{array}\right]}_{\mathbf{C}} \underbrace{\left[\begin{array}{c}
\mathbf{x}_{1}(n) \\
\mathbf{x}_{2}(n)
\end{array}\right]}_{\mathbf{x}(n)}
\end{gathered}
$$

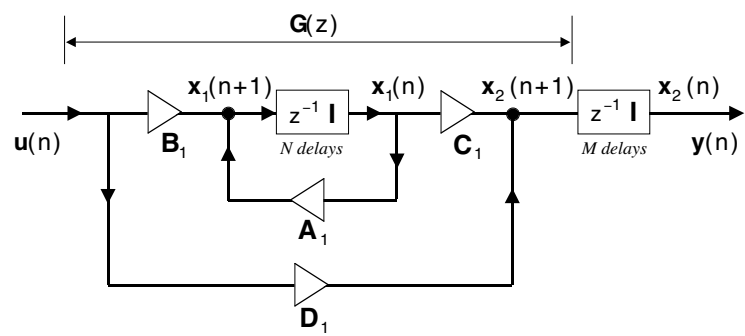

Figure 3. Details of the state space description of a cascade of $\mathbf{G}(z)$ with $z^{-1} \mathbf{I}$.

This shows that the matrices $(\mathbf{A}, \mathbf{B}, \mathbf{C}, \mathbf{D})$ in the state space description of Fig. 3 are

$$
\mathbf{A}=\left[\begin{array}{ll}
\mathbf{A}_{1} & \mathbf{0} \\
\mathbf{C}_{1} & \mathbf{0}
\end{array}\right], \quad \mathbf{B}=\left[\begin{array}{l}
\mathbf{B}_{1} \\
\mathbf{D}_{1}
\end{array}\right], \quad \mathbf{C}=\left[\begin{array}{ll}
\mathbf{0} & \mathbf{I}
\end{array}\right], \quad \mathbf{D}=\mathbf{0}
$$

\section{III.1. Observability}

We first examine the observability of the structure in Fig. 3. Recall from $\mathrm{PBH}$ test $[2,5]$ that $(\mathbf{C}, \mathbf{A})$ is not observable if and only if there exists a nonzero column-vector $\mathbf{v}$ such that

$$
\mathbf{A} \mathbf{v}=\lambda \mathbf{v}, \text { and } \mathbf{C v}=\mathbf{0}
$$

for some scalar $\lambda$. Using the form of $\mathbf{A}$ and $\mathbf{C}$ in Eq. (12) this can be rewritten as

$$
\left[\begin{array}{ll}
\mathbf{A}_{1} & \mathbf{0} \\
\mathbf{C}_{1} & \mathbf{0}
\end{array}\right]\left[\begin{array}{l}
\mathbf{v}_{1} \\
\mathbf{v}_{2}
\end{array}\right]=\lambda\left[\begin{array}{l}
\mathbf{v}_{1} \\
\mathbf{v}_{2}
\end{array}\right], \quad\left[\begin{array}{ll}
\mathbf{0} & \mathbf{I}
\end{array}\right]\left[\begin{array}{l}
\mathbf{v}_{1} \\
\mathbf{v}_{2}
\end{array}\right]=\mathbf{0}
$$

This can only be satisfied if $\mathbf{v}_{2}=\mathbf{0}$ (see second equation above). Thus (C, $\mathbf{A})$ is not observable if and only if there exists a nonzero column-vector $\mathbf{v}_{1}$ such that

$$
\mathbf{A}_{1} \mathbf{v}_{1}=\lambda \mathbf{v}_{1} \text {, and } \mathbf{C}_{1} \mathbf{v}_{1}=\mathbf{0}
$$

But this violates observability of $\left(\mathbf{C}_{1}, \mathbf{A}_{1}\right)$, thereby contradicting minimality of $\left(\mathbf{A}_{1}, \mathbf{B}_{1}, \mathbf{C}_{1}, \mathbf{D}_{1}\right)$. Thus $(\mathbf{C}, \mathbf{A})$ is necessarily observable.

This result is also clear by inspection of the structure. Thus, from measurements of $\mathbf{y}(n)$, the state vector $\mathbf{x}_{2}(n)$ is trivially identified. Since $\mathbf{x}_{2}(n+1)$ is the output of the first system, its identifiability means that $\mathbf{x}_{1}(n)$ can be identified as well if the first system is observable.

\section{III.2. Reachability}

Reachability of $(\mathbf{A}, \mathbf{B})$ is more tricky. Recall from $\mathrm{PBH}$ test that this is not reachable if and only if there exists a nonzero column-vector $\mathbf{v}$ such that

$$
\mathbf{v}^{\dagger} \mathbf{A}=\lambda \mathbf{v}^{\dagger}, \quad \text { and } \quad \mathbf{v}^{\dagger} \mathbf{B}=\mathbf{0},
$$

for some scalar $\lambda$. Eq. (14) can be rewritten as

$$
\left[\begin{array}{ll}
\mathbf{v}_{1}^{\dagger} & \mathbf{v}_{2}^{\dagger}
\end{array}\right]\left[\begin{array}{ll}
\mathbf{A}_{1} & \mathbf{0} \\
\mathbf{C}_{1} & \mathbf{0}
\end{array}\right]=\lambda\left[\begin{array}{ll}
\mathbf{v}_{1}^{\dagger} & \mathbf{v}_{2}^{\dagger}
\end{array}\right]
$$

and

$$
\left[\begin{array}{ll}
\mathbf{v}_{1}^{\dagger} & \mathbf{v}_{2}^{\dagger}
\end{array}\right]\left[\begin{array}{l}
\mathbf{B}_{1} \\
\mathbf{D}_{1}
\end{array}\right]=\mathbf{0}
$$

From the first equation we get

$$
\mathbf{v}_{1}^{\dagger} \mathbf{A}_{1}+\mathbf{v}_{2}^{\dagger} \mathbf{C}_{1}=\lambda \mathbf{v}_{1}^{\dagger}, \quad \mathbf{0}=\lambda \mathbf{v}_{2}^{\dagger}
$$

The second equation above is possible only if either $\mathbf{v}_{2}=\mathbf{0}$ or $\lambda=0$. If $\mathbf{v}_{2}=\mathbf{0}$, then from Eq. (15), (16) we get

$$
\mathbf{v}_{1}^{\dagger} \mathbf{A}_{1}=\lambda \mathbf{v}_{1}^{\dagger}, \quad \mathbf{v}_{1}^{\dagger} \mathbf{B}_{1}=\mathbf{0}
$$

This violates the reachability of $\left(\mathbf{A}_{1}, \mathbf{B}_{1}\right)$ which contradicts the assumption that $\left(\mathbf{A}_{1}, \mathbf{B}_{1}, \mathbf{C}_{1}, \mathbf{D}_{1}\right)$ represent a minimal relization. Thus, the second equation of (17) can only be satisfied with $\lambda=0$ in which case Eq. (15) yields

$$
\left[\begin{array}{ll}
\mathbf{v}_{1}^{\dagger} & \mathbf{v}_{2}^{\dagger}
\end{array}\right]\left[\begin{array}{l}
\mathbf{A}_{1} \\
\mathbf{C}_{1}
\end{array}\right]=\mathbf{0}
$$

Combining this with (16) we conclude that $(\mathbf{A}, \mathbf{B})$ is not reachable if and only if

$$
\left[\begin{array}{ll}
\mathbf{v}_{1}^{\dagger} & \mathbf{v}_{2}^{\dagger}
\end{array}\right]\left[\begin{array}{ll}
\mathbf{A}_{1} & \mathbf{B}_{1} \\
\mathbf{C}_{1} & \mathbf{D}_{1}
\end{array}\right]=\mathbf{0}
$$

where the vector $\left[\begin{array}{ll}\mathbf{v}_{1}^{\dagger} & \mathbf{v}_{2}^{\dagger}\end{array}\right]$ is nonzero. Equivalently $(\mathbf{A}, \mathbf{B})$ is not reachable if and only if

$$
\left[\begin{array}{ll}
\mathbf{A}_{1} & \mathbf{B}_{1} \\
\mathbf{C}_{1} & \mathbf{D}_{1}
\end{array}\right]
$$

is singular. Now, it has been shown in [6] that the above matrix is singular if and only if $\mathbf{G}(z)$ does not have an anticausal inverse. Thus, even when $\left(\mathbf{A}_{1}, \mathbf{B}_{1}\right)$ is reachable, the pair $(\mathbf{A}, \mathbf{B})$ is unreachable when $\mathbf{G}(z)$ does not have a anticausal inverse. Summarizing these discussions on reachability and observability we arrive at the following: 
Theorem 1. Cascading with delays. Let $\mathbf{G}(z)$ be an $M \times$ $M$ system with a minimial realization $\left(\mathbf{A}_{1}, \mathbf{B}_{1}, \mathbf{C}_{1}, \mathbf{D}_{1}\right)$. Consider the new system $\mathbf{H}(z)=z^{-1} \mathbf{G}(z)$ which can be implemented by adding $M$ delays as in Fig. 3. This implementation is minimal if and only if $\mathbf{G}(z)$ has an anticausal inverse, or equivalently, if and only if the matrix (19) is nonsingular. Thus

$$
\operatorname{deg}\left[z^{-1} \mathbf{G}(z)\right]=M+\operatorname{deg}[\mathbf{G}(z)]
$$

if and only if $\mathbf{G}(z)$ has an anticausal inverse. $\diamond$

\section{Example 1: Paraunitary systems}

If $\mathbf{G}(z)$ is a $M \times M$ causal paraunitary system [5], then its inverse

$$
\mathbf{G}^{-1}(z)=\left[\mathbf{G}^{*}\right]^{T}\left(1 / z^{*}\right)
$$

is anticausal. For example, the causal paraunitary system

$$
\mathbf{G}(z)=\frac{1}{2}\left[\begin{array}{ll}
1+z^{-1} & 1-z^{-1} \\
1-z^{-1} & 1+z^{-1}
\end{array}\right]
$$

has the anticausal inverse

$$
\mathbf{G}^{-1}(z)=\frac{1}{2}\left[\begin{array}{ll}
1+z & 1-z \\
1-z & 1+z
\end{array}\right]
$$

Thus, for paraunitary $\mathbf{G}(z)$, the degree of $z^{-1} \mathbf{G}(z)$ is exactly $N+M$, where $N$ is the degree of $\mathbf{G}(z)$.

\section{Example 2: System with no Anticausal Inverse}

Return to the example $\mathbf{G}(z)$ shown in Fig. 2(a). The state space matrices for $\mathbf{G}(z)$ are

$$
\mathbf{A}_{1}=0, \quad \mathbf{B}_{1}=\left[\begin{array}{ll}
1 & 1
\end{array}\right], \quad \mathbf{C}_{1}=\left[\begin{array}{l}
1 \\
1
\end{array}\right], \quad \mathbf{D}_{1}=\left[\begin{array}{ll}
0 & 0 \\
0 & 0
\end{array}\right]
$$

so that

$$
\left[\begin{array}{ll}
\mathbf{A}_{1} & \mathbf{B}_{1} \\
\mathbf{C}_{1} & \mathbf{D}_{1}
\end{array}\right]=\left[\begin{array}{lll}
0 & 1 & 1 \\
1 & 0 & 0 \\
1 & 0 & 0
\end{array}\right]
$$

This matrix is singular because the last two columns are identical. So it has no anticausal inverse. The the conditions of the theorem are violated, and the degree of $z^{-1} \mathbf{G}(z)$ is less than $N+M=1+2=3$. Indeed the degree is only two, as seen from Fig. 2(c).

\section{Example 3: Unimodular systems}

Next consider

$$
\mathbf{G}(z)=\left[\begin{array}{cc}
1 & 0 \\
z^{-1} & 1
\end{array}\right]
$$

This is a degree one unimodular matrix, and its inverse is the causal system

$$
\mathbf{G}^{-1}(z)=\left[\begin{array}{cc}
1 & 0 \\
-z^{-1} & 1
\end{array}\right]
$$

Since the unique inverse is causal, there is no anticausal inverse and we conclude that the degree of $z^{-1} \mathbf{G}(z)$ is necessarily less that $N+M=3$.

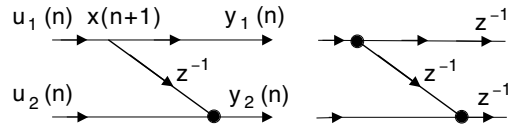

(a)

(b)

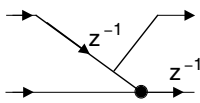

(c)
Figure 4. (a) Example of a unimodular system $\mathbf{G}(z)$ with degree one, (b) the cascaded system $\mathbf{H}(z)=z^{-1} \mathbf{G}(z)$ with two extra delays, and (c) structure for $\mathbf{H}(z)$ with mimimum delays.

A minimal implementation of $\mathbf{G}(z)$ is shown in Fig. 4(a). Note that

$$
\mathbf{H}(z)=z^{-1} \mathbf{G}(z)=\left[\begin{array}{cc}
z^{-1} & 0 \\
z^{-2} & z^{-1}
\end{array}\right]
$$

and its "obvious" implementation shown in Fig. 4(b) has three delays. This is indeed not minimal because there is an implementation with two delays (Fig. 4(c)). For completeness let us also check out the state space matrices. The state space description of the implementation of $\mathbf{G}(z)$ in Fig. 4(a) is

$$
\mathbf{A}_{1}=0, \quad \mathbf{B}_{1}=\left[\begin{array}{ll}
1 & 0
\end{array}\right], \quad \mathbf{C}_{1}=\left[\begin{array}{l}
0 \\
1
\end{array}\right], \quad \mathbf{D}_{1}=\left[\begin{array}{ll}
1 & 0 \\
0 & 1
\end{array}\right]
$$

so that

$$
\left[\begin{array}{ll}
\mathbf{A}_{1} & \mathbf{B}_{1} \\
\mathbf{C}_{1} & \mathbf{D}_{1}
\end{array}\right]=\left[\begin{array}{lll}
0 & 1 & 0 \\
0 & 1 & 0 \\
1 & 0 & 1
\end{array}\right]
$$

This matrix is singular (the first and last columns are identical), consistent with the fact that $\mathbf{G}(z)$ has no anticausal inverse.

\section{CONCLUding REMARKS}

The result revisited in Sec. II is central to the many factorization theorems for paraunitary filter banks proved in [5]. An interesting problem would be to extend the results of Sec. II for the case of noncausal systems which require both delay and advance operators in general. It will also be interesting to see how the results of Sec. III can be extended to the case of $P \times M$ systems with $P \neq M$, and to cascades of the form $\boldsymbol{\Lambda}(z) \mathbf{G}(z)$ where $\boldsymbol{\Lambda}(z)$ is a diagonal matrix whose diagonal elements are possibly unequal delays.

\section{REFERENCES}

[1] Y. Inouye, and R-W. Liu, "A system-theoretic foundation for blind equalization of an FIR MIMO channel system"IEEE Trans. on Circuits and Systems -I, pp. 425-436, April 2002.

[2] T. Kailath, Linear systems, Prentice Hall, Inc., 1980.

[3] A. V. Krishna, and K. V. S. Hari, "Filter bank precoding for FIR equalization in high-rate MIMO communications", IEEE Trans. on Signal Processing, pp. 1645-1652, May 2006.

[4] Y-P. Lin and S-M. Phoong, "Minimum redundancy for ISI free FIR filter bank transceivers", IEEE Trans. on Signal Processing, pp. 842-853, April 2002.

[5] P. P. Vaidyanathan, Multirate systems and filter banks, Prentice Hall, Inc., 1993.

[6] P. P. Vaidyanathan and T. Chen, "Role of anticausal inverses in multirate filter banks - I: System-theoretic fundamentals", IEEE Trans. on Signal Processing, pp. 1090-1102, May 1995. 